Revue d'histoire de l'Amérique française

REVUE D.HISTOIRE DE L'AMÉRIQUE FRANÇAISE

\title{
Un seigneur éclairé, Louis-Joseph Papineau
}

\section{Roger Le Moine}

Volume 25, numéro 3, décembre 1971

URI : https://id.erudit.org/iderudit/303090ar

DOI : https://doi.org/10.7202/303090ar

Aller au sommaire du numéro

Éditeur(s)

Institut d'histoire de l'Amérique française

ISSN

0035-2357 (imprimé)

1492-1383 (numérique)

Découvrir la revue

Citer cet article

Le Moine, R. (1971). Un seigneur éclairé, Louis-Joseph Papineau. Revue

d'histoire de l'Amérique française, 25(3), 309-336.

https://doi.org/10.7202/303090ar d'utilisation que vous pouvez consulter en ligne.

https://apropos.erudit.org/fr/usagers/politique-dutilisation/ 


\title{
UN SEIGNEUR ÉCLAIRÉ, LOUIS-JOSEPH PAPINEAU *
}

\author{
ROGer LE MOINE \\ Université d'Ottawa
}

En 1802, le notaire Joseph Papineau reçut du Séminaire de Québec, comme remboursement des sommes qui lui étaient dues, les trois cinquièmes de la seigneurie de la Petite-Nation ${ }^{1}$. Deux ans plus tard, il se porta acquéreur de l'autre partie de ce fief, devenant ainsi l'un des grands propriétaires terriens du pays ${ }^{2}$. La Petite-Nation mesurait cinq lieues de front, sur la rivière Outaouais, par autant de profondeur - environ 15 milles carrés ou 180,000 arpents -, superficie fort importante, même à l'époque ${ }^{3}$. Cette seigneurie bénéficiait de plusieurs privilèges accordés par l'acte de concession. Louis-Joseph Papineau les énumère dans une lettre à sa femme:

Elle ne contient pas l'obligation de concéder ni de le faire à un taux fixe. Elle ne fait aucune réserve ni de bois ni de mines de quelque nature qu'elles soient pour la couronne mais au contraire les abandonne au seigneur. Elle n'est pas sujette au droit de Quint en cas de vente. Elle a donc ce me semble tous les avantages de la franche tenure. Elle ne doit à la couronne que la foi et hommage de 20 en 20 ans si elle est demandée et aussi à chaque mutation de Roi ou de

Papineau.

* L'année 1971 marque le centenaire de la mort de Louis-Joseph

** Sigles et abréviations: AFB, Archives de la famille Bourassa; APC, Archives publiques du Canada; APQ, Archives de la province de Québec; FD, Fonds Dessaulles; FPB, Fonds Papineau-Bourassa; L.-J.P., Louis-Joseph Papineau; M.-B., Monte-Bello; N.B., Napoléon Bourassa; P., Papineau; S.M.E., Séminaire de Québec.

1 Administrateur pour le S.M.E. de la seigneurie de l'lle Jésus, Joseph Papineau avait fait construire à ses frais des moulins dont on n'avait pu lui rembourser le coût autrement qu'en lui abandonnant les $3 / 5$ de la Petite-Nation. Cette seigneurie avait été concédée en 1674, par la Compagnie des Indes occidentales, à Mgr de Montmorency-Laval qui l'avait léguée, de même que tous ses autres biens, au S.M.E., par un acte passé à Paris devant les notaires De Troy et Carnot, le 12 avril 1680, puis ratifié à Québec, le 16 mai 1681, par le notaire Rageot.

2 Fernand Ouellet, Papineau, dans les Cahiers de l'Institut d'histoire (Québec, P.U.L., sd), 99.

3 Loc. cit.

RHAF, vol. 25, no 3 (décembre 1971) 
Seigneur si elle est demandée et dans la pratique elle ne l'est pas et à chaque prestation de la foi et hommage, elle doit en tout et pour tout payer un anneau d'or ou sa valeur fixée à 11 livres tournois un peu plus de dix chelins sterling. C'est une charge purement nominale pour constater la Suzeraineté de la couronne non pour le profit. ${ }^{4}$

En 1810, Joseph Papineau construisit dans l'île à Roussin ${ }^{5}$ une maison où sa famille vécut pendant quelques années. Il y concéda quelques terres où des colons commencèrent le défrichement. Quatre ans plus tard, le curé de Sainte-Geneviève, l'abbé Dumouchel, célébra une première messe chez les Papineau ${ }^{6}$. Par la suite, et jusqu'à la fondation de la paroisse, les missionnaires de l'Outaouais et de la Gatineau prirent l'habitude de s'arrêter chez le seigneur. Dès l'année 1815, Mgr Plessis avait demandé au supérieur des Sulpiciens d'envoyer de temps à autre un religieux de sa communauté dans ces parages. L'abbé Jean-Baptiste Roupe fut le premier à répondre à cette invitation et, en août 1815, il passa dix-neuf jours à la Petite-Nation ?

Le 2 mai 1817, Joseph Papineau céda à son fils LouisJoseph, pour la somme de 5,000 louis, la majeure partie de sa seigneurie ${ }^{8}$. L'arrière-fief de Plaisance, créé à ce moment, revint à un autre de ses fils, Denis-Benjamin, qui s'établit sur son bien vers $1820^{9}$; et, jusqu'en 1837, c'est chez ce dernier que Louis-Joseph descendait lorsqu'il séjournait dans la région. Le nouveau seigneur avait alors 31 ans ${ }^{10}$. Il était député depuis 1808 et il pratiquait le droit depuis 1810 . Il avait fait l'acquisition, en 1814, de la maison de son père, rue Bonsecours ${ }^{11}$, puis il avait épousé, le 23 avril 1818, Julie Bruneau, la fille

${ }^{4}$ L.-J. Papineau à sa femme, Paris, 15 juillet 1845, dans $R A P Q$, 36-37 (1955-1957): 302.

5 Robert Rumilly, Papineau (Montréal, Valiquette, s.d.), 12.

6 Note d'Adine B., APQ, FPB, boîte 88.

7 Loc. cit.

8 Loc. cit. (Greffe du notaire Louis Guy).

9 Loc. cit.

10 J.-J. Lefebvre, "La vie sociale du grand Papineau", dans $R H A F$, 9, no 4 (mars 1958) : 469.

11 Joseph Papineau était né dans une maison de bois sise au même endroit. Il la remplaça par une maison de pierre plus petite que la maison actuelle, qu'il vendit puis reprit avant de s'en départir au profit de son fils, vraisemblablement au moment où il alla s'établir dans l'île à Roussin. Après avoir loué la maison, de 1829 à 1831, L.-J. P. la restaura, l'agrandit à son tour et s'y installa. Lui et sa famille cessèrent de l'occuper en 1837. $\mathrm{Au}$ retour de l'exil, ils n'y séjournèrent que quelques semaines. 
d'un marchand et député de Québec, Pierre Bruneau. Dix enfants devaient naître de ce mariage ${ }^{12}$.

En se portant acquéreur de la Petite-Nation, Papineau voulait débarrasser son père d'un fardeau trop lourd pour son âge. En 1817, Joseph Papineau avait 65 ans et il n'était plus en mesure d'entreprendre de vastes travaux d'exploitation. De plus, il avait conscience d'effectuer un bon placement: les spéculations immobilières auxquelles se livraient certains seigneurs provoquaient déjà une hausse du prix des terres arables et obligeaient les fils de cultivateurs à s'établir sur des lots nondéfrichés et plus éloignés comme ceux de la Petite-Nation. Mais, en dépit de la conjoncture favorable, le nombre des colons n'augmenta d'abord que lentement. Une douzaine de maisons à peine sont construites en 1820 et $1821^{13}$. Six ans plus tard, la situation n'ayant guère changé, Papineau écrit à son frère Denis-Benjamin :

Que dis-tu de l'idée d'encourager l'établissement d'Irlandais catholiques? Ils sont ici d'un merveilleux accord avec les Canadiens. Je voudrais pourtant que ceux-ci se portassent en plus grand nombre chez moi. ${ }^{14}$

Le seigneur faisait bien tout son possible pour remédier à la situation. Dès 1820 , il avait contribué de ses deniers à la construction d'une chapelle qui, placée sous l'égide de Notre-Damede-Bonsecours (comme la paroisse de Montréal à laquelle les Papineau appartenaient), avait été bénite le 3 septembre 1821, en présence de plusieurs membres de la famille ${ }^{15}$. Et le nouveau seigneur se disait prêt à se charger de l'entretien du curé pourvu que l'évêque lui apportât quelque aide ${ }^{16}$. Pour récompenser tous ces efforts, l'évêque de Montréal, Mgr Lartigue, qui était le cousin germain du seigneur, procéda, le 26 septembre 1831, à

12 Ce sont: Amédée (1819-1903), Didier (1820-1821), Lactance (18221862), Ernest (1823- ?), Arthur (1824-1825), Aurélie (1826-1830), Ezilda (1828-1894), Gustave (1829-1851) et Azélie (1834-1869). De plus, Madame Papineau fera une fausse couche en 1837, au moment où, menacée par les gens du Doric Club, elle dut s'enfuir de la maison de la rue Bonsecours avec ses cadets.

13 L.-J.P. à sa femme, Petite-Nation, 7 nov. 1821, APC, FP.

${ }^{14}$ L.-J.P. à Denis-Benjamin P., Petite-Nation, 20 août 1827, APC, FP.

15 Les invités sont Mme Joseph Papineau; ses fils, l'abbé ToussaintVictor et Louis-Joseph, qui est accompagné de sa femme; son gendre, le seigneur Jean Dessaulles, de Maska; son beau-frère, Denis-Benjamin Viger et sa femme, Marie Amable Fortier. Notons encore la présence du docteur René-Joseph Kimbert et du notaire Jean-Joseph Girouard. (Note d'Adine Bourassa, loc. cit.)

16 L.-J.P. à Denis-Benjamin P., Petite-Nation, 20 août 1827, APC, FP. 
l'érection canonique de la paroisse et l'abbé Paisley fut nommé curé ${ }^{17}$.

En 1826, Papineau terminait la construction d'un moulin à scie qui lui coûta toujours très cher d'entretien ${ }^{18}$. Le moulin banal existait depuis plusieurs années déjà ${ }^{19}$. Malheureusement, les moyens de communication laissaient à désirer. Le transport du bétail restait difficile. Il n'y avait pas de route et, quant à la rivière, il fallait compter avec les rapides du Long-Sault ${ }^{20}$. Le Phenix, le Lady Simpson et le Breeze ne desservirent les établissements de l'Outaouais de façon régulière, entre Bytown et Sainte-Anne-de-Bellevue, que plusieurs années plus tard. Et, à Sainte-Anne, on était encore loin de Montréal.

Durant cette période, Papineau séjournait à la Petite-Nation deux fois par année. Il en profitait pour réclamer les cens et rentes, parfois sans succès. Il fait remarquer à sa femme, le 9 avril 1828:

Je ne pouvais ajouter à l'embarras qu'il y avait dans la famille, celui de remplir la maison de monde, en appelant les Censitaires. D'ailleurs, je vois que je n'en retirerai rien, il est trop tard, leurs grains sont mangés. ${ }^{21}$

De même, il faisait exécuter quelques travaux:

Je n'ai point arrêté quand il a fait beau: aller au Moulin de la Chûte inventorier les effets que je livre à $\mathrm{Mr}$ Mears, faire finir la maison au moulin de la baie, y placer une seconde paire de moulanges; faire sortir le fermier ...22

Comme il aimait mêler l'utile à l'agréable, il ne manquait pas l'occasion de se livrer à la pêche ${ }^{23}$ ou de se rendre à la cabane

17 Note d'Adine Bourassa, loc. cit.

18 Denis-Benjamin P. à L.-J.P., Petite-Nation, 5 déc. 1826, APC, FP. 19 L.-J.P. à sa femme, Petite-Nation, 7 nov. 1821, APC, FP.

20 Papineau écrit à sa femme, le 7 novembre 1821: "Je partirai jeudi pour t'aller revoir mais comme nous partirons en barge, que nous aurons affaire chez Mr Mears; que te menant comme tu le désires, une vache, il faudra la faire marcher dans le long sault et l'attendre au pied des rapides pour la reprendre dans le bateau, parce que la faire mener par terre coûterait les gages de son conducteur et qu'une aussi longue route pourrait la faire tarir, nous pourrions avoir des gros vents contraires durant lequel on ne marcherait pas, il ne faut pas que tu t'inquiètes si nous ne nous rendions que samedi ou dimanche." (Petite-Nation, APC, FP.) 249.

21 L.-J.P. à sa femme, Petite-Nation, 9 avril 1828, dans RAPQ, 34-35:

22 L.-J.P. à sa femme, Petite-Nation, 13 janvier 1822, dans RAPQ, 34-35: 202.

23 Loc. cit. L.-J.P. à sa femme, Petite-Nation, 7 nov. 1821, APC, FP. 
à sucre ${ }^{24}$. Papineau aurait aimé prolonger ses séjours dans la seigneurie. En homme d'affaires averti, il savait très bien qu'on ne peut administrer à distance une entreprise aussi considérable. Le 28 mai 1833, il écrit à sa femme:

Je suis dans un bel endroit mais bien pauvre et bien souffrant, en partie parce que nous n'y sommes pas. ${ }^{25}$

A l'époque, il songeait à établir dans la région un de ses enfants:

Le temps est venu où notre intérêt et celui de notre famille demandent que nous passions les étés dans un établissement qui deviendra très important. Quelqu'un de tes enfans, comme médecin, notaire ou marchand, serait dans le cas de s'établir à la campagne. ${ }^{26}$

Bien des événements se produiront avant que ce rêve ne se réalise. Malgré toute sa bonne volonté, le jeune seigneur était incapable, à l'époque, de s'établir à la Petite-Nation ou même d'y passer de longues vacances. Marié, père de famille, avocat, il s'était de plus lancé dans la carrière politique qui accaparait toutes ses énergies. De sorte que la seigneurie ne se développait pas comme il l'aurait voulu. Et c'est très justement qu'il écrivait en 1855:

Je l'acquis en 1817, et tout absorbé comme je fus par les tourments de la vie politique, je la négligeai et les progrès furent lents. ${ }^{27}$

Lorsque des problèmes urgents se posaient, Denis-Benjamin Papineau qui, comme on sait, demeurait tout près, essayait de trouver des solutions. Mais cette tâche devenait d'autant plus lourde au co-seigneur de Plaisance qu'il devait lui-même consacrer tout son temps à l'administration de ses propres biens. Denis-Benjamin ne continua pas moins de remplacer son frère, surtout pendant les huit années de l'exil (1837-1845). Car la Révolution ayant échoué, Papineau, qui comptait parmi les chefs des Patriotes, avait gagné les Etats-Unis et la France d'où il ne rentra qu'en 1845 .

Pendant son séjour à l'étranger, aux Etats-Unis ou en France, Papineau ne cessa pas de songer à son avenir. De quelle 371.

${ }^{24}$ L.-J.P. à sa femme, Petite-Nation, 9 avril 1828, dans RAPQ, 34-35:

25 L.-J.P. à sa femme, Petite-Nation, 28 mai 1833, APC, FP. 371.

26 L.-J.P. à sa femme, Québec, 10 décembre 1835, dans RAPQ, 34-35:

27 L.-J.P. à Eugène Guillemot, s.l., 10 janvier 1855, dans Fernand Ouellet, op. cit., 99. 
façon pourra-t-il assurer sa subsistance et celle de sa famille ? Voilà la première de ses préoccupations. Il écrit à son frère Denis-Benjamin :

Mon cher, il faut commencer par m'assurer et à ma famille bien modestement l'habit et la vie, et si tout ce que j'ai de ressources peut à peine suffire, à cette première nécessité il ne faut songer à rien autre chose. D'autres en peuvent souffrir avec moi. C'est un mal irrémédiable. C'est une considération additionnelle pour me forcer à chercher s'il est possible de vendre et de s'établir ailleurs, mais non un motif d'emprunter, pour voir vendre forcément et pour rien ailleurs des propriétés qui ont une grande valeur d'estimation. ${ }^{28}$

Pas question pour lui de se remettre à la pratique du droit. Mais s'il vendait la Petite-Nation, il pourrait vivre dans une certaine aisance ${ }^{29}$. Sans compter qu'il possédait à Montréal des biens immobiliers d'un bon rapport. Il passa par une période d'hésitations, se demandant s'il devait se retirer à Saratoga où, au moment des premières épreuves, il avait pu compter sur d'excellents amis et où il pourrait agir à sa guise, sans craindre les autorités britanniques. Il songea également à Saint-Hyacinthe où vivait sa chère sœur la seigneuresse Dessaulles. C'est dans l'espoir de trouver un acquéreur qu'au départ de Paris, en 1845, il s'arrêta à Londres. D'ailleurs, dès le mois de mars 1845, en prévision des pourparlers qu'il voulait entreprendre, il avait demandé qu'on lui transmette certains documents indispensables aux buts poursuivis ${ }^{30}$. Le 15 juillet de la même année, après un voyage en Italie, il déplore toujours que l'on n'ait pas donné suite à sa demande du mois de mars ${ }^{31}$. C'est dans la même lettre qu'il raconte ses pourparlers avec un acquéreur possible, John Arthur Roebuck, un député anglais qui, à l'époque des 92 résolutions, s'était fait le porte-parole des Canadiens français à la Chambre des Communes de Londres. Papineau raconte sa tentative en essayant de faire comprendre à sa femme que, s'il échoue, ce ne sera pas faute de bonne volonté.

Papineau compte également sur l'agent d'une compagnie anglaise qui séjournait au Canada. Le 25 avril 1845, il autorise son frère Denis-Benjamin à transiger en son nom, fixant le prix de vente de la seigneurie à 30,000 livres sterling $(\$ 120,000 \text {. })^{32}$.

28 L.-J.P. à Denis-Benjamin P., s.l., s.d., APC, FP.

29 L.-J.P. à sa femme, Paris, 1er mars 1845, dans RAPQ, 36-37: 287.

30 Loc. cit.

31 L.-J.P. à sa femme, Paris, 15 juillet 1845, dans RAPQ, 36-37: 300.

32 L.-J.P. à Denis-Benjamin P., Paris, 25 avril 1845, AFB. 
Pour des raisons que l'on ignore, l'affaire n'eut pas de suite. Le séjour de Londres n'ayant pas donné non plus les résultats escomptés, Papineau écrit à sa femme, le 18 août 1845 , qu'il en a pris son parti : la famille s'établira à la Petite-Nation ${ }^{33}$. Avant même les retrouvailles, une cause de discorde s'élevait entre les époux.

Julie Papineau, qui ne se plaisait pas sur les rives de l'Outaouais, refusait catégoriquement, à l'époque, de s'établir dans la seigneurie. Elle invoquait toutes sortes d'arguments si on en juge par les réponses de son mari - craignant entre autres de ne pouvoir, dans les forêts seigneuriales, trouver de maris à ses filles. Azélie et Ezilda partageaient facilement ses vues. Sans compter que Julie qui venait de retrouver parents et amis après un long exil, n'entendait pas les quitter de sitôt ${ }^{34}$.

En dépit de l'attitude de sa femme, Papineau resta inébranlable. D'ailleurs, il lui était presque impossible d'agir autrement. Sa situation financière n'était pas brillante. Le problème était le suivant: comment réussir à vivre sans tirer parti de la seigneurie ? Les allusions à la précarité des moyens de la famille sont nombreuses dans la correspondance de l'époque. Et tout nous porte à croire que seuls la maladie de Lactance et le désir d'établir ses fils firent fléchir Julie. Papineau note, dans une lettre à Pierre Margry, qu'il a entrepris ses travaux de colonisation "en vue d'utiliser pour eux [les enfants] bien plus que pour [lui], dans [son] age avancé, la vaste propriété qu'[il a] dans une campagne éloignée et peu habitée..." ${ }^{35}$. De toute façon, la seigneuresse ne se résigna jamais complètement à cette vie campagnarde. De là le compromis auquel ils arrivèrent. Madame Papineau et ses filles vivraient à la Petite-Nation l'été et une partie de l'automne, et elles quitteraient la seigneurie pour Montréal juste avant la fermeture de la navigation sur l'Outaouais. Papineau viendrait les retrouver peu avant Noël, effectuant le trajet en carriole. L'entente ne fut pas toujours observée et les Papineau passèrent parfois l'année entière à la Petite-Nation.

$\mathrm{Au}$ fond, Papineau n'était pas fâché de la tournure des événements. Il était attaché à cette terre où il avait séjourné

${ }^{33}$ L.-J.P. à sa femme, Londres, 18 août 1845, dans RAPQ, 36-37: 309.

34 L.-J.P. à sa femme, Genève, 20 juin 1845, dans RAPQ, 36-37: 296298. - L.-J.P. à sa femme, Londres, 18 août 1845, dans RAPQ, 36-37: 309.

35 L.-J.P. à Pierre Margry, Petite-Nation, 19 nov. 1852, dans Lettres $\grave{a}$ Pierre Margry de 1844 à $1886 \ldots$ présentées par Ls-P. Cormier (Québec, P.U.L., 1968), 2. 
aussi souvent qu'il avait pu. Et l'idée de jouer au grand propriétaire terrien, lui dont la famille n'était sortie de l'obscurité que depuis une génération, ne lui déplaisait pas. L'exemple de certains seigneurs français, qu'il avait connus, ne le laissait sans doute pas indifférent. Mais à ces considérations d'amour-propre s'en ajoutaient d'autres qui le déterminèrent à se lancer à fond dans cette entreprise. Comme Vigny, qu'il avait rencontré à Paris, il n'acceptait guère la nouvelle civilisation industrielle; il lui préférait une certaine forme de vie patriarcale dont le seigneur était le chef. Son opposition à l'abolition du régime seigneurial s'inscrit dans cette optique. Dans ses interventions à la Chambre d'assemblée, de 1848 à 1854, il fut beaucoup moins soucieux de défendre ses intérêts que certains historiens l'ont prétendu. Durant ses longues méditations parisiennes, il avait sans doute entrevu son nouveau rôle. Et, lorsque le cabinet Draper-Viger lui versa la somme de 4,500 livres sterling $(\$ 18,000)$ - son salaire d" "orateur" de la Chambre d'assemblée - il put passer du rêve à la réalité.

Papineau a joué, jusqu'au moment de son exil, un rôle prépondérant dans la société canadienne. Et il compte bien ne pas rester inactif, même à la Petite-Nation. Car, si l'ère des discours est passée, le vieux tribun ne poursuit pas moins les mêmes rêves humanitaires; il est animé du même désir d'aider ses compatriotes. Nourri des auteurs du XVIII ${ }^{\mathrm{e}}$ siècle, il n'eut pas à chercher sa voie bien longtemps; l'exemple du patriarche de Ferney s'offrait à lui. Marcel Trudel a établi, il y a déjà longtemps, un parallèle entre les deux hommes ${ }^{36}$. Leurs carrières respєctives présentaient de nombreuses similitudes. Tous deux avaient échoué dans leurs entreprises sociales et politiques; tous deux avaient été exilés. Papineau n'avait plus qu'à créer son Ferney et à prôner à ses censitaires cette morale du travail apprise dans la lecture de Candide. C'est animé de cet esprit qu'il pose les bases d'une cité nouvelle qui s'inspire aussi des vues des sociologues français du XIX ${ }^{\mathrm{e}}$ siècle.

Ne possédant pas de pied-à-terre dans la seigneurie, Papineau loue d'abord la maison du forgeron Fortin qu'il enjolive afin d'y attirer sa famille; et ce n'est pas sans arrière-pensée qu'il écrit à sa femme, le 26 octobre 1846 :

${ }^{36}$ Marcel Trudel, L'influence de Voltaire au Canada (Montréal, Fides, 1945), 2: 50 . 
J'ai profité du beau jour de mercredi pour faire arracher au jardin de mon frère [Denis-Benjamin], dans la baie, des vignes de France, lilas, boules de neige et rosiers et les planter autour de la maison de Fortin. ${ }^{37}$

Puis, les travaux préliminaires commencent par le défrichement d'un promontoire qui surplombe une courbe de l'Outaouais. C'est dans ce décor qu'il entend construire sa maison. Il note:

J'ai aussi fait faire plusieurs chemins et défrichemens de broussailles seulement et de bois secs et renversés, sur le Cap, de manière a y laisser un grand bocage d'arbres verts et d'arbres à feuilles caduques distribués en jolis massifs au milieu desquels je pourrai bâtir une grande maison en retirant partie de ce qui m'est dû et qui serait perdu s'il fallait attendre avec autant de patience comme on l'a fait, que les gens voulussent bien payer. ${ }^{38}$

Et il ajoute, toujours dans la même lettre:

J'ai fait creuser les caves de 64 pieds de long, du côté est et ouest, sur 44 pieds de large, nord et sud. Quand nous serons ensemble nous en tracerons les plans. Il n'y a dans cet endroit qu'un et demi à $2 \frac{1}{2}$ pieds de terre sur le roc vif. Dans le voisinage, pour jardin et verger, trois pieds et demi et plus. ${ }^{39}$

Quelques mois plus tard, soit le 5 novembre 1846, il précise qu'il construira en pierres:

Dis à Benjamin que je ne veux pas bâtir en briques. Qu'il n'y a rien à mon avis de moins raisonnable que de courir les risques d'une pareille expérimentation, quand on a mieux, de belles pierres à proximité et point chère. J'ai contracté pour celles-ci. ${ }^{40}$

Les travaux sont brutalement interrompus au printemps de 1847. Papineau doit se rendre à Saint-Hyacinthe, au chevet de son fils Gustave. Puis il revient à la seigneurie et, pendant l'été et l'automne qui suivent, il s'occupe de défrichement. Il note, dans une lettre à sa femme: "J'ai quarante hommes et une douz' de bœufs ou chevaux qui travaillent pour moi..." 41. Au printemps suivant, il s'occupe d'ensemencer les champs qui entourent

\footnotetext{
36-37: 310.

38 Loc. cit.

39 Ibid., 311. 314.

40 L.-J.P. à sa femme, Petite-Nation, 3 nov. 1846, dans RAPQ, 36-37: 36-37: 335 .
}

${ }^{37}$ L.-J.P. à sa femme, Petite-Nation, 26 octobre 1846, dans RAPQ, 
le promontoire. Les récoltes sont bonnes puisqu'à l'automne de 1848, il peut expédier à Montréal 40 minots d'avoine ${ }^{42}$. La construction reprend, quoiqu'au ralenti, en septembre 1849. A l'époque, les plans du manoir sont tracés de façon définitive. Amédée y avait collaboré avec ses sœurs et Julie avait donné son approbation. Un entrepreneur du nom de Dubucque avait tout revisé ${ }^{43}$. En novembre 1849, les travaux vont bon train ${ }^{44}$. L'été suivant, l'édifice n'est pas encore habitable puisque les Papineau logent dans la maison de Fortin ${ }^{45}$. Ils n'y entreront qu'en mai 1851 et l'acquisition du mobilier ne se fera que très lentement. Les derniers travaux ne seront effectués qu'en 1853. Il écrit, le 27 mai de cette année-là :

(...) l'on a monté les trois têtes de cheminées et fixé l'une à sa place, sans accident. C'est une grande chose, car quand il y a quelqu'opération aussi difficile que de remuer et porter si haut des blocs si lourds je les crains toujours. Les têtes de cheminées vont être réparées aujourd'hui et leurs couvertures en pierre placées, après quoi l'on travaillera à la balustrade. Ce sera je pense un bel embellissement à la maison. ${ }^{46}$

Le manoir terminé, Papineau construit, à une faible distance, une chapelle funéraire qui sera bénite par Mgr Guigues, en $1855^{47}$, et où il transporte les restes des membres de sa famille. Il érige également deux pavillons de brique près de la grille d'entrée. L'un logera le jardinier et l'autre servira de remise à grains ${ }^{48}$. Ensuite, il se lance dans la construction de la bibliothèque. Il écrit à Robert Christie, le 19 mai 1856:

Je me suis décidé à me bâtir une haute tour, détachée de la maison, mais assez rapprochée pour que, à quelque distance, elle en paraîtra comme une aile. Je la fais à l'abri du feu, pour mettre à couvert de ce risque, mes chers livres et le grand nombre de contrats \& de papiers à la conservation desquels tant de familles peuvent être intéressées dans la seigneurie. ${ }^{49}$

C'est à la même époque qu'il aménage les abords du manoir. Dans la correspondance, il ne cesse de commander des pommiers

${ }^{42}$ L.-J.P. à sa femme, Petite-Nation, 15 octobre 1848, dans RAPQ, 36-37: 336 .

43 L.-J.P. à sa femme, 30 sept. 1859, APC, FP.

44 L.-J.P. à sa femme, 1 er et 6 nov. 1849, dans RAPQ, 36-37: 340.

45 L.-J.P. à sa femme, s.l., 29 juin 1850, dans RAPQ, 36-37: 345-346.

46 L.-J.P. à sa femme, s.l., 27 mai 1853, dans RAPQ, 36-37: 353. Cette

"balustrade" sera peinte en jaune.

47 Note d'Adine Bourassa, APQ, FPB, boîte 88 .

48 Loc. cit.

49 L.-J.P. à Robert Christie, s.l., 19 mai 1856, AFB. 
de toutes variétés, des ceps de vigne, des rosiers...50. Pour plaire à ses deux filles, il prépare à chacune un petit jardin ${ }^{51}$ et il s'émerveille ensuite des résultats obtenus:

... les étoiles \& cercles coupés dans le gazon se couvrent de vervaines de toutes couleurs \& diverses autres fleurs; ça commence à être moins ébourrifé de roches et de souches qu'Amédée ne le désirera. ${ }^{52}$

La lettre qu'il adresse à Pierre Margry, en 1852, donne une idée assez exacte de l'importance des travaux réalisés:

J'ai commencé il y a quatre ans, au beau milieu de la forêt des défrichemens étendus, construction de maison d'habitation et dépendance, plantation de verger, et jardin Chinois pour ne pas dire Anglais. Les enfans voulaient quelque chose de mieux que ce qui se voit en Canada, parce que le local y sollicitait parce que nous qui avions vu tant de bon modèles, serions plus justement critiqués que d'autres, si nous pechions contre les regles du goût. Dans l'isolement du lieu, j'ai été architecte, agriculteur, dessinateur et jardinier. Obligé de tout diriger et surveiller, j'ai fatigué plus que ne le comportaient mon âge et les habitudes antérieures de la vie sédentaire d'étude et de bureau. Ma santé est affaiblie, chagrin: par ma faute, reproche; et ceux pour qui je travaillais, selon leur goût et à leur demande, finis avant moi hélas; finis avant ces travaux..$^{53}$

Ce sont des propos semblables qu'il tient à Eugène Guillemot, en 1855:

Ma femme avait toujours abhorré l'idée de vivre à la campagne et pour tâcher de surmonter ses répugnances, je songeai à un établissement plus beau qu'il n'était strictement nécessaire de le faire; et pour accélérer sa complétion, je m'exposai à trop de fatigues. J'ai été architecte, le dessinateur des vergers et jardin, le défricheur du terrain. J'ai eu pendant trois été entiers au-delà de cinquante travailleurs de tous métiers à diriger et j'ai à répondre aujourd'hui à plus de quatre cents colons, chefs de famille et propriétaires de lots de terre depuis vingt arpents, à douze ou quinze cents selon leurs facultés. ${ }^{54}$

50 L.-J.P. à Amédée P., Petite-Nation, 23 mars 1862, APC, FP.

51 L.-J.P. à sa femme, s.l., 5 juin 1853, dans RAPQ, 36-37: 358.

52 L.-J.P. à sa femme, Petite-Nation, 20 mai 1853, APC, FP.

53 L.-J.P. à Pierre Margry, Petite-Nation, 19 novembre 1852, dans Lettres à Pierre Margry ..., op. cit., 2.

54 L.-J.P. à Eugène Guillemot, s.l., 10 janvier 1855, dans Fernand Ouellet, Papineau, op. cit., 100. 
Il ne restait plus qu'à baptiser le nouveau domaine. Cela s'imposait, le gouvernement voulant établir dans la seigneurie un second bureau de poste auquel il fallait trouver un nom. Après avoir suggéré à sa famille les noms de Montfleury, Mont-Joly, Montalto, Belmont, Beaumont et Monte-Bello, il opte pour ce dernier ${ }^{55}$.

En exploitant sa seigneurie, Papineau veut d'abord faire vivre les siens dans une certaine aisance, celle dont ils ont l'habitude. Cette préoccupation ne l'empêche pas de songer également à ses compatriotes. Car, s'il érige un moulin banal et un moulin à scie, s'il met tant de soins à importer des arbres fruitiers, à planter son verger, à ensemencer son potager, s'il fait l'essai de nouvelles techniques agricoles - il serait intéressant de dresser l'inventaire des traités d'agriculture contenus dans sa bibliothèque - c'est pour donner l'exemple à ses censitaires, envers qui il se sent obligé, et pour les aider du mieux qu'il peut à améliorer leur condition. On aimerait posséder la correspondance qu'il échangea avec eux. Ainsi pense-t-il leur faciliter l'accès au bonheur. Monte-Bello, c'est une ferme modèle.

A force d'efforts, les concessions augmentent. En 1855, 400 colons et leurs familles se sont déjà établis à la Petite-Nation. Dans les 4 ans qui suivent, 200 autres viennent les rejoindre ${ }^{56}$. Ce mouvement oblige le seigneur, qui n'est plus jeune, à ouvrir des rangs et à tracer les rues et avenues d'un village qui prendra, il l'espère, les proportions d'une petite ville:

[Je] me suis résolu d'y avoir un village; me suis fait arpenteur pour le tracer en rues larges de 60 pieds, bordée d'arbres, obligé les acheteurs à en garnir le front des lots que je vends, chaque rue portant les noms des arbres qui y sont plantés dans toute leur longueur. Ainsi mon village devient-il comme Londres; on s'y reconnaîtra toujours facilement, les noms des rues étant indiqués par les arbres qu'elles porteront. J'ai

55 Note d'Adine Bourassa, APQ, FPB, boîte 88. Le nom doit s'écrire en deux mots si l'on en croit Henri Bourassa: "Et je demande aux journalistes d'écrire Monte-Bello en deux mots. Un abbé a soutenu qu'il fallait l'écrire en un seul mot parce que Louis-Joseph Papineau avait fait amitié avec le duc de Montebello et qu'il avait appelé le manoir en souvenir de ce personnage. C'était très ingénieux, mais c'était faux; Papineau a fait écrire Monte-Bello en deux mots pour que ce soit différent du nom du duc." (Henri Bourassa, "Qui était cet homme ?", dans Hommage à Henri Bourassa (Montréal, Imprimerie populaire), 37. Henri Bourassa était mieux renseigné que l'abbé Michel Chamberland, l'auteur de l'Histoire de Montebello, à qui il est fait allusion ici. D'ailleurs, Papineau l'écrivait en deux mots.

56 Fernand Ouellet, Papineau, op. cit., 100. 
déjà des lots sur des rues: des ormes, érables, tilleuls, chênes, etc, et des pins, sapins, sapinettes cèdre. Les rues courant de l'est à l'ouest, arbres à feuilles caduques, celles qui les coupent à angles droits vont du sud au nord avec arbres à feuilles persistantes. Il est aisé de s'orienter. J'ai retiré des terrains adjoignants la fabrique, à la charge de lui bâtir maison et chapelle. ${ }^{57}$

Le présent permet d'envisager le futur avec enthousiasme.

Le seigneur ne peut se contenter d'apporter à ses censitaires le bien-être matériel. Il tente également de les délivrer de l'ignorance. C'est dans ce but qu'il invite les Sœurs Grises d'Ottawa à venir s'installer dans la seigneurie, offrant même aux religieuses un terrain où bâtir le couvent. La requête de Papineau ayant été favorablement reçue, sœur Ste-Thérèse et sœur Sainte-Anne répondent à l'appel et arrivent le 6 septembre $1867^{58}$. Elles ne furent pas accueillies avec une égale sympathie par toute la population! Bien involontairement, elles prenaient la place du maître d'école qui soudoya ses amis. Prenant prétexte d'une possible augmentation des taxes, " 4 à 5 peres de famille enfonc[ent] avec barres de fer chassis et portes d'une maison" où les sœurs avaient commencé d'enseigner. Ils s'attaquent ensuite au presbytère, forçant cinq fenêtres et intimant au curé l'ordre de déguerpir dans les huit jours ${ }^{59}$. Ils voyaient ce dernier comme l'un des responsables de la venue des religieuses. Pour mettre un terme à tous ces excès, Papineau dut faire appel à la police de Montréal. Le calme ne fut rétabli qu'à la minovembre ${ }^{60}$.

Tout en étant extrêmement satisfait de son expérience seigneuriale, Papineau, qui avait été habitué à un théâtre autrement plus vaste, déplore le caractère limité de son action; elle ne dépassait pas les limites de la Petite-Nation. Il croit qu'en ces années où le pays passe par des moments cruciaux, il peut encore jouer un rôle utile. Les offres nombreuses qui lui sont faites de part et d'autre rendent la tentation irrésistible. Il se décide de passer à l'action et se présente, en 1848, dans le comté de Saint-Maurice puis, en 1852, dans le comté des Deux-Montagnes où les Patriotes avaient fait preuve de tant de bravoure.

${ }^{57}$ L.-J.P. à Robert Christie, s.l., 19 mai 1856, AFB. Assez malencontreusement, ces rues ont été rebaptisées de noms de saints.

58 Sœur Paul-Emile, Mère Elisabeth Bruyère et son œuvre. Les Scurs Grises de la Croix (Ottawa, Editions de l'Université, 1945), I: 321.

59 L.-J.P. à Amédée P., M.-B., 10 octobre 1857, AFB.

60 Sœur Paul-Emile, Mère Elisabeth Bruyère ..., loc. cit. 
L'esprit de la Confédération règne déjà. Les propos qu'il tient à Mgr Guigues, à la fin de son mandat, expriment son désenchantement:

Serez-vous encor à Québec lors de l'ouverture du Parlement ? Je conviens de bon cœur que la réunion du concile sera toute fructueuse, édifiante et désirée par tout le monde tandis que la nôtre, comme d'accoutumé, aura ses scandaleuses animosités et tant et tant de stériles discussions qu'elle est plutôt redoutée que désirée. Mais enfin, tel est l'ordre providentiel du monde politique. Il est livré aux vaines disputes des discoureurs. $\mathrm{Si}$, au milieu du profond ennui que me donne à mon âge l'agitation de la vie publique, j'ai le bonheur de vous rencontrer et de vous rendre mes devoirs, j'y trouverai un bon moment de consolation. S'il m'est donné de pouvoir être de quelque utilité à des établissements aussi utiles que ceux que vous fondez, je le désirerai avec empressement. ${ }^{61}$

Etre utile, voilà encore à quoi il tient. Et la politique le lui interdit pendant un certain temps.

Durant cette période de sa vie, Papineau passe la plus grande partie de l'année à la Petite-Nation. Généralement, il ne séjourne à Montréal que pendant les mois de janvier et de février. Mis à part ce voyage annuel, il ne s'éloigne de la seigneurie que lorsqu'il est forcé de le faire, soit à cause de la politique, soit pour des raisons familiales. Il fera le voyage de New York au moment où, en 1846, Lactance sera hospitalisé à Bloomingdale ${ }^{62}$. La grave maladie dont souffre Gustave l'oblige à passer les mois de février, mars et avril 1847 à Saint-Hyacinthe ${ }^{63}$. C'est dans l'espoir de voir Azélie se remettre de ses malaises qu'il séjourne à Philadelphie, durant l'hiver de $1857^{64}$.

Puis, les deuils et les déchirements succèdent aux inquiétudes. Gustave meurt le 17 décembre $1851^{65}$. L'année suivante, Lactance s'en va finir ses jours à Saint-Jean-de-Dieu de Lyon ${ }^{66}$. Puis disparaissent des parents auxquels il est également très attaché, comme Denis-Benjamin Papineau, en 1854, Rosalie

${ }^{61}$ L.-J.P. à Mgr Guigues, Petite-Nation, 15 mai 1854, APC, FP.

62 L.-J.P. à sa femme, New York, 20 novembre 1846, dans RAPQ, 36-37: 315-316.

63 L.-J.P. à sa femme, s.l., 17 mars 1857, AFB. Sur la maladie de Gustave et sur celle de son frère Lactance, voir Lionel Groulx, "Fils de grand homme", dans RHAF, 10, no 3 (décembre 1956): 329 sqt. De même, Mes Mémoires (Montréal, Fides, 1971), II : 229-248.

64 Azélie P. à sa mère, s.l., 17 mars 1857, AFB.

65 Lionel Groulx, "Fils de grand homme", op. cit., 329.

66 Ibid., 330. 
Papineau-Dessaulles, en 1857, l'abbé Toussaint-Victor Papineau, en 1869, Madame Denis-Benjamin Papineau (Angèle Cornud), en juillet 1870, Louis-Michel et Denis-Benjamin Viger en 1855 et 1861. Ajoutons encore à cette kyrielle, d'autres tracas domestiques. Les récoltes ne sont pas toujours bonnes, les censitaires ne versent pas les rentes régulièrement. Et il s'inquiète de ce que son gendre Napoléon Bourassa exerce un art qui ne lui permette pas de faire vivre sa famille.

Les petits-enfants, surtout ceux d'Azélie qu'il voyait plus fréquemment, lui font oublier les vicissitudes de l'existence. Et, peu après la naissance de Gustave Bourassa, il écrit à Amédée une lettre tout imprégnée de la pensée de Candide:

Et bien oui mon cher Amédée, malgré les nombreuses mésaventures de Candide, je m'en tiens un peu aux doctrines du sage docteur Pangloss et du Grand Leibnitz, qu'il serait impie de ne pas croire que nous sommes dans le meilleur des mondes possible, qu'il est impossible que nous ne soyons pas dans le meilleur des mondes possibles, autrement Dieu n'aurait pas fait comme il en avait bonne envie. Il y a bien par ci, par là des momens, où l'on croirait que le Diable l'emporte on ne sait où, pour le briser on ne sait comment. Tant tout y va de travers, mais cela n'est pas une raison suffisante de conclure contre une démonstration démonstrative. Tu comprends, que ceux qui sont vieux radottent; et je ne suis pas jeune quand je crois si fort en Pangloss et Leibnitz. C'est chère Azélie, elle était moins hier, sinon toujours, dans le meilleur des mondes possibles. ${ }^{67}$

Mais avec quel autre membre de sa famille, Papineau aurait-il pu causer de la philosophie de Pangloss et parodier un texte de Voltaire?

Qu'il se trouve à Monte-Bello ou à Montréal, Papineau est toujours entouré du même cercle de parents. Ce sont sa femme, ses filles Azélie et Ezilda, et son gendre Napoléon Bourassa. Il se sent exilé, lui, l'homme du XVIII ${ }^{\mathrm{e}}$ siècle, croyant mais non pratiquant, au milieu de ce "parti des dévôts" qu'est sa famille. Sans doute, l'un des grands drames de son existence fut de constater que les idées pour lesquelles il s'était battu, auxquelles il continuait de croire, n'étaient partagées que par l'un de ses enfants, Amédée. La constatation est amère. Madame Papineau avait inculqué à ses deux filles les principes religieux de l'époque. Dans l'idée de sauver de la damnation le chef de la famille, elles multipliaient les pratiques de dévotion, courant les retraites

${ }^{67}$ L.-J.P. à Amédée P., M.-B., 16 juin 1860, AFB. 
paroissiales jusqu'à Verchères, Saint-Denis-sur-Richelieu et Saint-Hyacinthe. Tous ces excès de zèle exaspéraient le vieillard, car il saisissait fort bien les motifs qui animaient les trois femmes. Quant à Bourassa, formé comme il l'avait été par les maîtres de l'Ecole mystique allemande, il défendait les idées de Mgr Bourget. Et en jouant un rôle au sein de plusieurs sociétés bien pensantes, il luttait contre ce à quoi son beau-père tenait par-dessus tout: la liberté et la tolérance. Curieux retour des choses, quand on songe que Papineau faisait vivre son gendre !

On imagine facilement l'atmosphère qui régnait dans les soirées familiales. Chacun tentait d'éviter les sujets de controverse. De sorte qu'on était forcé de s'en tenir à la politique étrangère - sauf celle de l'Italie - aux potins, à l'aménagement de la seigneurie. Parfois, la conversation portait également sur les arts, et particulièrement sur la musique et la peinture. Papineau lisait beaucoup. Il s'intéressait aux grands événements qui se déroulaient au pays et à l'étranger. On retrouve dans ses lettres de l'époque, surtout dans celles qu'il adressait à Amédée ou au neveu Louis-Antoine Dessaulles, des jugements empreints de sagesse, de lucidité, et révélateurs de ses idées politiques et religieuses.

Durant les dernières années de sa vie, Papineau devait être éprouvé par d'autres deuils aussi cruels que les précédents. Sa femme meurt subitement, le lundi 18 août 1862 , à sept heures du matin. Voici comment il raconte la scène à Mrs. John Keyes Paige, dans cette belle lettre où il évoque toute sa carrière:

She slept a quiet night, when she awoke at 6 with horrid cries suffering tortures, she said, between the shoulders. All were nears in the minute. She exclaimed "Be certain that such pains are more than human strenght can bear. It cannot last long. Send at once for the priest and physician", which was done, but in vain. The priest near at hand absolved her. The physician live at 4 miles distance, and thought he arrived within an hour's time, life was extinct. We knew it not; thought of syncope, used frictions, salt and burned feathers in vain. The physician had to declare death, owing to effusion of blood to the hearth. ${ }^{68}$

Ezilda et Azélie perdent non seulement une mère, mais aussi une confidente et une amie qui avait toujours su comprendre leurs problèmes et intervenir auprès de son mari quand elle le jugeait à propos. C'est à leur sujet que le père écrit qu'elles

${ }^{68}$ L.-J.P. à Mrs. John Keyes Paige, Montréal, 30 décembre 1864, AFB. 
sont "jeunes d'âge mais vieillies dans la peine, les pertes, les morts, la vie errante et la maladie de notre pauvre Lactance" ${ }^{69}$.

La maladie et la mort imprévue d'Azélie, survenue le 27 mars 1869, n'affectent pas moins Papineau. D'autant plus qu'entre le décès de Julie et celui d'Azélie, Lactance s'était éteint à Lyon, le 4 décembre 1862, sans avoir revu les siens. De cette famille de dix enfants, seuls survivaient le père, Amédée et Ezilda.

Papineau, qui ne veut pas laisser prise au découragement, se remet à la tâche. Comme par le passé, il tente de pratiquer la morale de Zadig et de Candide. Dans une lettre à Amédée, où il fait part de sa règle de vie, on retrouve encore une fois les influences qui s'exercèrent sur lui :

Notre part dans la vie n'est probablement que la quote-part moyenne qui échoit à ceux qui y sont appelés. Soyons soumis à porter avec résignation ses mécomptes, à goûter avec modération ses jouissances, tout cela est bien insuffisant à nos désirs. Mais les désirs plus ardents trompent si souvent ceux qui les ont qu'ils doivent rendre plus malheureux que cette tiède et timide philosophie ne le fera. Puis, au-delà du passage, obscurité insoluble. C'est là ce qui empoisonne la vie des oisifs et des penseurs et n'effleurera que légèrement celle de ceux qui se donnent de sages et utiles occupations travaillons donc! 70

"Travaillons sans raisonner, disait le Martin de Candide, c'est le seul moyen de rendre la vie supportable." Le bonheur et la sérénité ne se trouvent que dans le travail et la modération. Voilà la philosophie qui orientait sa vie. On retrouve les mêmes idées dans cette lettre à Mrs. Paige que nous avons déjà citée.

La lecture avait également sur Papineau des effets apaisants. C'est vers la même époque qu'il confie au curé Médard Bourassa:

L'étude, toujours, c'est l'une des plus efficaces distractions aux plus poignantes douleurs. J'achette tous les jours des livres et perds beaucoup de tems à lire les contemporains. $\mathrm{Au}$ travail, il y a tou'ours plus de médiocre que d'excellent. A étudier le passé, l'on ne lit que l'excellent qui a survécu. ${ }^{71}$

Travail, étude, modération, voilà de quoi était fait son art de vivre.

${ }^{69}$ L.-J.P. à Médard Bourassa, Montréal, 5 juin 1864, APC, FP.

70 L.-J.P. à Amédée P., s.l., 10 oct. 1857, AFB.

71 L.-J.P. à Médard B., Montréal, 5 janvier 1863, AFB. 
Papineau aimait faire visiter son domaine. Il en était fier. Les gens venaient de partout pour voir le manoir, les dépendances, les parterres, les potagers, les vergers, la vigne, les champs. C'était l'oasis, au milieu du désert. Il prit froid au début de septembre 1871, alors qu'il déambulait dans les allées du parc en compagnie d'un visiteur français. La pneumonie se déclara. Après être passé par des moments critiques, son état s'améliora. La famille se mit à espérer un rétablissement et Napoléon Bourassa put écrire à Fanny Leman-Dessaulles, le 20 septembre 1871:

Il me semble que nous pouvons avoir plus d'espoir; la journée a été bien meilleure qu'on pouvait l'espérer ce matin; pour moi, le mieux est sensible, la respiration de notre cher malade est plus libre, il a pu reposer dans son lit durant quelques heures, ce qu'il n'avait pas fait depuis dimanche; il y a quelque chose de plus normal dans le fonctionnement du cœur et par la suite dans la circulation. Le moral se relève aussi. Enfin, il me semble qu'il y a dans tout ceci de quoi faire, pour vous, une heureuse nouvelle et je me hâte de vous l'adresser parce que vous en avez besoin.

Eh bien, quoi que cette maladie soit de nature à ébranler fortement sa constitution, aujourd'hui, j'ai plus espoir qu'il va s'en relever encore, avec quelque vigueur. Il est si étonnamment constitué. ${ }^{2}$

Trois jours plus tard, l'état du malade inspire de nouvelles craintes. Bourassa précise, à propos de l'attitude religieuse de Papineau, à ce moment:

Vous savez sans doute déjà, que votre pauvre oncle a refusé tout secours religieux; il a déclaré au curé "ne pas croire dans la Révélation, n'y avoir jamais cru, il confie avec calme son éternité à la justice d'un Dieu bon et juste". Je vous adresse ce détail parce que je sais qu'il doit être une cause d'inquiétude parmi les siens. Mais il serait mieux de le garder tout à fait entre nous, pour le moment. ${ }^{73}$

Dans une lettre à P.-N. Pacaud, Louis-Antoine Dessaulles confirme les propos de Bourassa, tout en révélant des détails que la première lettre ne contient pas:

Le curé de l'endroit, frère de son gendre, s'est conduit en digne prêtre et en homme sensé, il est allé lui offrir ses services et mon oncle lui a dit qu'il le voyait toujours avec plaisir, comme ami de la famille, et comme son ami personnel, mais qu'il ne pouvait, sans mentir à Dieu et aux

${ }^{72}$ N.B. à Fanny Leman, M.-B., 20 septembre 1871, AFB.

73 N.B. à Joseph Leman, M.-B., 23 septembre 1871, AFB. 
hommes, le recevoir comme prêtre; qu'il ne croyait pas à la révélation; qu'après cinquante ans d'étude et de méditations sérieuses sur ces matières, il en était venu à la conclusion que toutes les religions avaient une somme de bien et de très belles choses à côté d'une certaine somme de faux et de superstitions; que toutes prétendaient à la vérité exclusives et qu'aucune ne la possédait plus que les autres; qu'ainsi tout en croyant aussi invinciblement que personne à l'existence de Dieu et aux devoirs moraux des hommes, il n'admettait pas les cultes, que les hommes avaient chargés de tant de superstitions, que sa conscience le forçait de désavouer, qu'il voyait venir la mort avec un calme absolu, persuadé que Dieu ne le punirait pas pour n'avoir cru à des dogmes qu'en son ame et conscience il croyait injurieux à sa bonté et à sa justice. Le curé lui observa très amicalement, que son exemple pourrait avoir de bien funestes conséquences dans le pays, et qu'un mot de lui, quand ce serait pas une profession de foi explicite pourrait aussi avoir un grand effet pour le bien.

Voudriez-vous donc, mon cher curé, que j'arrive devant Dieu avec un mensonge à la bouche, et une hypocrisie devant les hommes, voulez-vous qu'en mourant, je perde ma propre estime en protestant de sentiment que je n'ai pas dans le cœur ? Je n'ai jamais pendant ma vie déguisé ma pensée, et voudriez-vous que je la déguise au moment de paraître devant Dieu? C'est dans ce moment-ci plus que jamais, mon cher curé, que je ne saurais mentir à Dieu et aux hommes. Si je me trompe, c'est de bonne foi, et je compte sur la miséricorde de mon Créateur. ${ }^{74}$

C'est dans ces dispositions qu'il s'éteignit, dans la soirée du 23 septembre 1871. Au cours de l'après-midi, il s'était fait porté près de la fenêtre de sa chambre donnant du côté du nord-ouest, d'où il pouvait voir une partie de ses jardins, bordés par les flots de l'Outaouais. C'est l'image qu'il emporta avec lui.

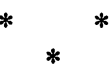

On devait croire dans certains milieux que Papineau reviendrait à la pratique du catholicisme au moment de mourir, qu'il s'en était éloigné temporairement à cause de l'attitude du clergé à l'époque de la Révolution de 1837. C'était, d'une façon malhonnête, mettre en doute la sincérité de l'homme. Mais au temps de Mgr Bourget, la mentalité avait été à ce point déformée qu'on doutait de la respectabilité de ceux qui n'acceptaient pas les dogmes et n'assistaient pas, chaque dimanche, à la grandmesse. A vrai dire, Papineau avait pu donner des espoirs au

${ }^{74}$ L.-A. Dessaulles à P.-N. Pacaud, 29 septembre 1871, APC, FD. 
parti dévôt. Son attitude est assez curieuse quand elle est vue de l'extérieur. Il avait agi parfois comme s'il avait ressenti la nostalgie de tout l'appareil de la religion. Il avait fait construire une église, fait vivre le curé, au début tout au moins, érigé une chapelle funéraire dans laquelle il avait placé un autel; il avait participé aux processions de la Fête-Dieu, assisté souvent à la messe et aux vêpres. Ses relations avec Mgr Guigues et le curé Bourassa avaient été très cordiales. Et, quand des membres de sa famille avaient été gravement malades, il n'avait pas hésité à mander un prêtre. Pourrions-nous affirmer, devant ces faits, qu'il ait été sensible, comme Chateaubriand, aux cérémonies du culte ? Sans doute pas dans une colonie aussi reculée que Monte-Bello. On ne pourrait pas soutenir davantage qu'il ait voulu donner l'exemple de la dévotion aux seules fins d'être suivi par son peuple ou de ne pas être volé par des gens qui croyaient aux peines de l'enfer. A n'en pas douter, Papineau avait beaucoup emprunté aux Philosophes, mais pas ce calcul mesquin ${ }^{75}$.

A force de lectures et de méditations, il avait fini par trouver la voie qui convenait à son tempérament. Dans son Discours devant l'Institut canadien, le 17 décembre 1867, discours qui est son testament spirituel, il avait noté:

Ce n'est que par le libre examen que l'on peut acquérir des convictions assez fermes pour qu'elles deviennent, en matière importante, une véritable foi très ardente, dont on veut la propagation et le triomphe à quelques risques et à quelques désagréments personnels qu'elle puisse nous exposer. ${ }^{76}$

Cette démarche accomplie, il avait rejeté les religions comme autant de foyers d'intolérance et de superstition. Marqué comme il l'avait été par les écrivains des Lumières, il n'aurait pu réclamer les secours de la religion sans voir se dérouler devant lui toute l'histoire de l'Inquisition. Papineau aurait volontiers souscrit à ce passage des Lettres sur le Canada d'Arthur Buies:

Je devais voir au Canada, en plein dix-neuvième siècle, autant d'indignité monstrueuse, autant d'absurdités que l'histoire en rapporte au Moyen-Age, moins les supplices, les autodafés, les tribunaux ecclésiastiques, toujours ruisselants de sang ou de larmes. ${ }^{77}$

$\mathbf{7 5}$ Voltaire a raisonné de cette façon, notamment dans les Dialogues entre $A, B$ et $C$ (17e entretien), et dans le Dictionnaire philosophique, à l'article "Enfer".

76 "Discours de l'Hon. L. J. Papineau (devant l'Institut canadien, le 17 déc. 1867.)", dans l'Annuaire de l'Institut canadien, 1867 (Montréal, Le Pays, 1868), 25.

77 Arthur Buies, Lettres sur le Canada (Montréal, l'auteur, 1864), 22. 
Mais, comme il avait vécu parmi des catholiques, qu'il n'avait pas voulu les blesser par son attitude, il s'était parfois prêté aux exercices du culte. Et c'était peut-être sa façon à lui de rendre hommage au clergé, dont il avait reconnu les mérites, pour le rôle joué dans la colonisation de l'Outaouais et de la Gatineau, et auprès de certains membres de sa famille. Quant aux arguties des "mites philosophiques" et des "animalcules à bonnets carrés", pour reprendre les expressions de Voltaire, il n'avait guère pu y souscrire. Selon le texte de la lettre de Bourassa que nous avons déjà citée, Papineau avait remis son salut à un "Dieu juste et bon". Ces épithètes, qui sont celles de Voltaire, expriment toute la métaphysique et toute la philosophie du seigneur de la Petite-Nation. Car l'homme d'action est porté à mépriser ces vains exercices de l'esprit humain; il sait bien que les systèmes n'ont jamais rassasié un ventre affamé. Maurice d'Orange-Nassau eut un mot qu'il aurait bien volontiers repris à son compte, s'il l'avait connu. Au moment des guerres de religion, le fils du Taciturne écrivit: "Je ne me soucie guère de savoir si la prédestination est verte ou bleue." Seule comptait pour Papineau l'action dans la tolérance. Sa seule et unique foi, il la porta au progrès, ce en quoi il était également de son siècle. Et quelles grandes choses n'attendait-il pas de l'Amérique:

Si ce qui se fait aujourd'hui est mieux que ce qui s'est fait dans le moyen âge, des traces de barbarie ne se pourront voir qu'en Europe, non en Amérique. Elle s'établit à une époque de science profonde, de goût exquis \& sur; l'Eclectisme n'y laissera naître et croître que ce qui est parfait. L'Avenir est à ce continent, voué par son isolement à la paix perpétuelle et à toutes les conquêtes de la paix, dans un âge intelligent. Voilà comme par la force de l'imagination, fermant tristement les yeux sur tout ce qui nous entoure, plongeant nos regards bien loin en avant, nous nous consolons de tout ce qui nous manque, en imaginant pour la postérité un merveilleux avenir de jouissances et de succès en tout genre. ${ }^{78}$

Les prophètes sont rares, même chez les philosophes. Souvent, l'admiration que l'on a pour un pays pousse à présumer de l'avenir en se basant sur le passé. Pourtant, où il y a histoire, il y a nécessairement barbarie.

La mort de l'honorable Louis-Joseph Papineau, ancien orateur de la Chambre d'assemblée et seigneur de la Petite-Nation, ne prit pas de temps à se répandre. De même en fut-il de ses dispositions ultimes. Et la réaction du milieu ultramontain fut

${ }^{78}$ L.-J.P. à Pierre Margry, Petite-Nation, 19 nov. 1852, dans Lettres d Pierre Margry ..., op. cit., 3 . 
prompte à se manifester. Si l'on en croit Louis-Antoine Dessaulles, l'illustre défunt fut condamné du haut de la chaire, dès le 8 octobre, par le sulpicien Antoine Giband, alors vicaire à Notre-Dame de Montréal:

Son sermon sur mon oncle, le 8 oct: 71, Com: est-il mort cet hom: Si illustre par l'intelligence et le savoir ? Sans foi, sans vouloir se reconnaître ! Où est-il aujourd'hui ? Combien d'entre vous ont peut-être envié sa haute position et l'influence qu'il s'était acquise dans le pays ? Et bien, qui d'entre vous voudrait être à sa place; qui voudrait aujourd'hui échanger son sort pour le sien? 79

Ce sont là des propos qui ne déshonorent que leur auteur ! D'ailleurs, ils n'ont rien d'original. Le procédé est connu !

Tous ceux qui avaient pris part aux luttes politiques de la première moitié du $\mathrm{XIX}^{\mathrm{e}}$ siècle - il en restait encore parmi ceux qui avaient eu 20 ou 30 ans en 1837 - voyaient disparaître celui qui avait assumé les revendications de tout un peuple et donné une direction précise à ses destinées. Pour Ezilda, Bourassa et ses enfants, cette disparition du chef de la famille en qui tous avaient su trouver aide et réconfort, marquait la fin d'une époque. Elle signifiait aussi la fin des séjours au manoir seigneurial. Car ils ne s'entendaient guère avec le nouveau seigneur, Amédée, qui avait hérité du manoir. Mais ils n'avaient pas été déshérités pour autant.

Louis-Joseph Papineau avait rédigé son dernier testament (olographe) le 20 octobre 1867, révoquant "tous testament et codicilles antérieurs [s'] arrêtant à celui-ci seulement" ${ }^{80}$.

A la vérité, ce testament n'est guère révélateur de l'état de la fortune du seigneur de la Petite-Nation. Tout au plus permetil de conclure que Papineau s'est montré juste et équitable envers ses enfants. Qu'il avantage Amédée n'était que légitime. Ne serait-il pas le chef de la famille ? Le document nous permet en outre d'affirmer que son auteur n'avait pas tellement confiance dans les capacités administratives de ses héritiers. C'est la raison pour laquelle il ne leur accordait que l'usufruit et la jouissance des biens. Reste à voir si les petits-enfants seraient mieux doués.

79 Cahier de L.-A. Dessaulles, 24, APC, FD. Et Dessaulles ajoute: "M. Giband soigné par un médecin de Montréal, pourri de vérole (Prof. Bibaud)."

${ }^{80} \mathrm{Le}$ testament fut enregistré au greffe du palais de justice de Montréal le 30 septembre 1871 . 
Pour se faire une idée exacte de la fortune de Papineau, il faut recourir au Partage des biens de la succession de l'Honorable L.J. Papineau entre ses héritiers testamentaires, long document de quatre-vingt-sept pages daté du 4 septembre 1878 et préparé par le notaire Denis-Emery Papineau (fils de DenisBenjamin), grâce au rapport des experts qui s'étaient vu confier la tâche d'inventorier les biens meubles et immeubles du défunt. A Monte-Bello, le travail fut effectué par François Marcotte et Louis-Dominique Fortin, un des hommes de confiance de Papineau, et à Montréal, par Joseph Laramée, entrepreneur, PierreHubert Morin et Pierre Giguère, tous deux inspecteurs d'immeubles. Voici le bilan auquel ils arrivèrent et que, par souci de clarté, nous présentons de façon schématique:

\section{A - Biens à partager d'après leur valeur estimative:}

"Mobilier corporel" gardé par Ezilda, c'est-à-dire le mobilier de la maison de Montréal.

$\$ 1,023.10$

"Mobilier corporel" gardé par Amédée, c'est-à-dire le mobilier du manoir.

La créance Dagenais

$\$ 2,204.00$

Les rentes constituées Appleton

$\$ 2,000.00$

Les créances dues par Amédée $\$ 4,000.00$

$\$ 1,760.00$

Les obligations du New York Central: trois de $\$ 1,000$, et deux de $\$ 500$.

Les indemnités seigneuriales

$\$ 4,000.00$

Il s'agit de "deux rentes constituées (...) crées et établies par le Cadastre de la Seigneurie "La Petite-Nation" fait par le commissaire pour l'abolition de la tenure seigneuriale Henry Judah Ecr icelles rentes constituées dues par le Gouvernement de la Puissance du Canada pour rachat des droits casuels et de banalité de moulin dans la dite seigneurie et payables semi-annuellement les premiers janvier et juillet de chaque année savoir I-une somme annuelle de mille cinquante et une piastres et treize piastres et quatre-vingthuit centins pour droit de lods et ventes $(\$ 17,528.88)$. II-Pour le droit de banalité de moulin, une autre somme annuelle de cent vingt piastres égale en capital à celle de deux mille piastres".

$\$ 19,528.88$

Rentes constituées seigneuriales des paroisses de Notre-Dame-de-Bonsecours [Monte-Bello] et de Sainte-Angélique [Papineauville] moins les côtes des Cascades et du Moulin. "Les rentes constituées dont il est question (...) sont établies en vertu du Cadastre de la Seigneurie La Petite-Nation, fait par Henry Judah Ecr l'un des Commissaires nommés pour mettre à l'effet les dispositions de l'Acte Seigneurial de 1854 et ses amendements."

$\$ 24,437.162 / 3$

Les rentes constituées seigneuriales de Saint-André-Avellin et des côtes des Cascades et du Moulin.

$\$ 25,062.331 / 3$

La maison paternelle (de la rue Bonsecours)

$\$ 10,000.00$ 
La maison de la rue Saint-Denis

$\$ 12,000.00$

L'emplacement du moulin

$\$ 600.00$

Le terrain du "Carré" Saint-Louis

$\$ 300.00$

Le domaine seigneurial ( $3^{\mathrm{e}}$ et $4^{\mathrm{e}}$ partie)

$\$ 9,920.00$

Total

$\$ 116,871.48$

B - Biens à partager d'après leur superficie en nature:

Selon l'Inventaire, ce sont les "terres de la dite succession tant celles situées dans les diverses côtes de la Seigneurie et non occupées des tiers, que celle du Franc alleu Papineau [terres exemptes de droits seigneuriaux], non encore mises en côtes ou concessions ouvertes, à la colonisation, [qui] seraient comprises et passées dans le présent partage, en nature, d'après leur superficie, arpent par arpent, telles qu'elles se trouvent sans plus de détail". Les motifs qui poussent les bénéficiaires à ne pas évaluer ces terres en argent sont données dans l'Inventaire: "Les terres dépendant de la dite seigneurie $(. .$.$) sont d'égales valeurs les unes portant les autres et peuvent$ se partager également et suivant les droits des dits héritiers du défunt." Ces terres sont situées soit dans le franc-alleu, soit dans Saint-André-Avellin. Nombre d'arpents

$11,372.30$

C-Terres sous bail-vente:

"Ce sont des terres (...) appartenant [aux héritiers], dont ils ont permi l'occupation par nombre de personnes. à certaines charges et conditions avec promesse de leur vendre les dites terres, si telles personnes leur payaient le prix convenu, et que seulement alors titre de vente serait donné: autrement que les argents donnés et payés ne seraient considérés que comme loyer des terrains occupés."

Terres sous bail-vente libres de rentes constituées 4,085 arpents

Terres sous bail-vente grevées de rentes constituées

et 87 perches

3,524 arpents

et 55 perches

(Ces rentes constituées, déià comprises dans les totaux, rapportaient, pour un capital de $\$ 2323.00$, des intérêts de $\$ 139.38$.)

$\mathrm{Au}$ total, les héritiers se partagèrent des biens évalués à $\$ 116,871.48$ et des terres d'une superficie de 18,982 arpents et 172 perches qui valaient environ $\$ 40,000$. En effet, c'est au prix de $\$ 2.25$ l'arpent que les Bourassa offrirent en vente les mêmes terres, une dizaine d'années plus tard. De sorte qu'on peut évaluer les biens de Papineau, au moment de sa mort, à environ $\$ 150,000$.; encore faut-il ajouter une somme de $\$ 45,000$. que les héritiers retirèrent du Syndicat de rachat des rentes, en 1940, au moment de l'abolition définitive des rentes seigneuriales constituées ${ }^{81}$.

81 Jean-Jacques Lefebvre, "La vie sociale du grand Papineau", dans la Revue d'histoire de l'Amérique française, 11, no 4 (mai 1957): 515. 


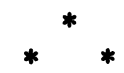

Le seigneur de la Petite-Nation agit-il en seigneur éclairé, c'est-à-dire, ses rapports avec les censitaires furent-ils marqués par les auteurs du XVIII ${ }^{\mathrm{e}}$ siècle ? C'est se lancer dans une entreprise assez hasardeuse que de répondre à cette question, car ceux qui se sont réclamés de l'idéal des Lumières ont conservé, assez souvent, leur originalité propre. De sorte que les attitudes varient selon les individus. Essayons quand même de dégager quelques constantes.

L'esprit philosophique consiste - nous recourrons d'abord à des témoignages - à "découvrir la raison qui fait que les choses sont et qu'elles sont plutôt ainsi que d'une autre manière" (L'Encyclopédie) ${ }^{82}$. C'est encore l'attitude de celui qui " $n$ 'admet rien sans preuve, [qui] n'acquiesce point à des notions trompeuses, [qui] pose exactement les limites du certain, du probable, du douteux" (L'Encyclopédie). Si Helvétius met l'accent sur cet "esprit d'observation et de justesse qui rapporte tout à ses véritables principes" ${ }^{83}$, Chamfort voit dans "le philosophe (...) un homme qui oppose la nature à la loi, la raison à l'usage, sa conscience à l'opinion et son jugement à l'erreur" 84. Pour La Motte, le philosophe doit revendiquer le "droit d'examiner" 85. Et nous pourrions prolonger cette liste - bien inutilement puisque les quelques exemples que nous avons apportés, permettent de voir se profiler la silhouette du philosophe, c'est-à-dire de celui qui veut détruire le préjugé et l'esprit d'autorité au profit de la raison, de l'observation et de l'expérience. Ces nouveaux critères de la connaissance incitent les philosophes - qui veulent percer le mystère des choses - à se livrer à une revision des notions fondamentales concernant le destin de l'homme et l'organisation de la société. Rejetant bientôt les solutions théologiques ou métaphysiques de même que l'autorité de la Tradition, ils substituent à la religion qui, selon eux, s'apparente à la superstition, un nouvel idéal, celui du progrès et du bonheur sur la terre. Comme l'écrit Robert Mauzi dans L'idée du bonheur dans la littérature et la pensée françaises du $X V I I I^{e}$ siècle:

82 V.-L. Saulnier, La littérature française du siècle philosophique (Paris, P.U.F., 1963), 7-8.

83 Ibid., 8.

84 Loc. cit.

85 Loc. cit. 
La pensée du XVIII' siècle découvre, pour la première fois peut-être que l'existence de l'homme ne se suffit pas à ellemême et réclame une justification. $\mathrm{Si}$ la condition humaine devient une énigme et un sujet d'angoisse, c'est que nul ne se sent plus soutenu par la stabilité de l'univers théologique du XVII ${ }^{e}$ siècle, et qu'il n'est plus de Révélation pour renseigner d'emblée chaque homme sur sa destination. ${ }^{86}$

Si l'on peut reprocher à quelques-uns un épicurisme facile, on doit reconnaître que ceux qui se contentèrent d'un juste bien-être sont plus nombreux. Conscients de la misère d'autrui, ils s'appliquèrent à la soulager et virent dans le progrès un remède à ce vice de la société qui, à cause du remords qu'il suscite, empêche le philosophe, l'homme éclairé, d'être heureux. Ces quelques paragraphes du Philosophe, ce texte du XVIII ${ }^{\mathrm{e}}$ siècle dont on n'a pu identifier l'auteur avec certitude, confirment notre avancé:

Le philosophe est donc un honnête homme qui agit en tout par raison, et qui joint à un esprit de réflexion et de justesse les mœurs et les qualités sociables. (...) On voit par tout ce que nous venons de dire, combien s'éloignent de la juste idée du philosophe ces indolents qui, livrés à une méditation paresseuse, négligent le soin de leurs affaires temporelles et de tout ce qui s'appelle fortune. Le vrai philosophe n'est point tourmenté par l'ambition; mais il veut avoir les douces commodités de la vie; il lui faut, outre le nécessaire précis, un honnête superflu nécessaire à un honnête homme, et par lequel seul on est heureux; c'est le fond des bienséances et des agréments.

La pauvreté nous prive du bien-être qui est le paradis du philosophe; elle bannit loin de nous toutes les délicatesses sensibles et nous éloigne du commerce des honnêtes gens.

A la vérité, nous n'estimons pas moins un philosophe pour être pauvre; mais nous ne le bannissons pas de notre société, s'il ne travaille à se délivrer de sa misère. Ce n'est pas que nous craignions qu'il nous soit à charge: nous l'aiderions dans ses besoins; mais nous ne croyons pas que l'indolence soit une vertu.

La plupart des hommes qui se font une fausse idée du philosophe s'imaginent que le plus exact nécessaire lui suffit: ce sont les faux philosophes qui ont fait naître ce préjugé par leur indolence et par des maximes éblouissantes. C'est toujours le merveilleux qui corrompt le raisonnable: il y a des sentiments bas qui ravalent l'homme au-dessous même de la pure animalité; il y en a d'autres qui semblent l'élever au-dessus de lui-même. Nous condamnons également les uns

86 Robert Mauzi, L'idée du bonheur dans la littérature et la pensée françaises au XVIII ${ }^{e}$ siècle (Paris, Colin, 1960), 51. 
et les autres, parce qu'ils ne conviennent point à l'homme. C'est corrompre la perfection d'un être que de le tirer hors de ce qu'il est, sous prétexte même de l'élever. ${ }^{87}$

Après cette approche assez sommaire de la définition du philosophe, nous tenterons de répondre à la question que nous formulions plus haut: Papineau appliqua-t-il, dans ses rapports avec les censitaires, la politique de l'homme éclairé ?

L'installation de Papineau à la Petite-Nation a été diversement interprétée. Les uns y ont vu le geste d'un homme politique déçu, qui décide de tourner le dos au monde, à la société qui n'a pas su le comprendre, en se réfugiant dans une retraite somptueuse, à la mesure de la haute opinion qu'il a de lui-même. D'autres ont tenté de démontrer que cette entreprise en peuplement n'était qu'une façon de s'enrichir, en pressurant ses censitaires. Non, Papineau n'était ni rancunier ni avide. Et dans les pages qui précèdent, nous avons essayé de montrer qu'en s'établissant à la Petite-Nation, il a voulu assurer sa subsistance et celle de sa famille, tout en aidant ses compatriotes sur un théâtre forcément plus restreint que jadis. Là, il a recherché le bonheur grâce au travail et au progrès. Et l'occasion était idéale. Car l'homme politique qui rentre d'exil sait maintenant - son expérience le lui a appris de même que ses lectures et ses méditations - qu'aucune grande œuvre n'est possible au milieu des convulsions de la politique et de la guerre.

Seigneur éclairé, Papineau le fut-il ? La réponse, pensonsnous, est affirmative. En autant que les adeptes des Lumières se sont interrogés sur la destinée et ont trouvé leur bonheur en tentant de le procurer aux autres. Sans doute Papineau, qui avait été marqué par le jansénisme et le rigorisme du milieu canadien-français, de même que par le puritanisme protestant, n'a pas donné dans cet épicurisme, dans ce sensualisme dont on trouve les échos dans le roman et la poésie du XVIII ${ }^{\mathrm{e}}$ siècle. Il aurait condamné les agissements des personnages de Laclos, de Prévost, et il n'aurait pas souscrit à telle strophe des Stances à Mme Lullin. Mais il avait compris, comme les esprits les plus perspicaces et les plus généreux du XVIII ${ }^{\mathrm{e}}$ siècle, que le bonheur ne s'acquiert que dans la mesure où l'on tente de le procurer aux autres, ne serait-ce qu'en leur apprenant les vertus fécondes du travail. Le bonheur ne saurait être égoïste. Nul doute que,

87 "Le Philosophe", Texts and Interpretation by Herbert Dieckmann (Washington University Studies, Saint-Louis, 1948), 58-62. Nous empruntons ce texte à la fois à la version publiée dans l'Encyclopédie et dans les Nouvelles libertés de penser. 
dans son domaine, il se préoccupa du sort de ses censitaires en essayant, plus que les autres seigneurs du temps ne le faisaient, d'élever leur niveau matériel et intellectuel. Inutile de rappeler encore une fois son action. Sans doute certains esprits assez bornés lui ont-ils reproché d'avoir exigé les cens et rentes, c'est-àdire son dû. Pourquoi se serait-il montré si généreux ? Parce qu'il avait sacrifié plusieurs années de sa vie à la politique ? Parce que la Révolution l'avait presque ruiné ? C'est là un triste raisonnement. C'eût été de la mégalomanie. Reproche-t-on à Voltaire ses habitudes de luxe ? D'ailleurs, pour faire vivre sa famille et, en même temps, poursuivre son action, il devait disposer de revenus assez considérables. La Petite-Nation exigeait de grands investissements, son exploitation n'ayant commencé qu'au XIX ${ }^{e}$ siècle. Non, les disciples des philosophes du XVIII ${ }^{\circ}$ siècle demandaient aux maîtres de jouir d'un "honnête superflu" qui permette à leurs obligés de connaître le bien-être, pour ne pas dire l'aisance. Voilà ce qui fut, semble-t-il, la ligne de conduite de Papineau. E'le concorde tout à fait avec les passages du Philosophe dans lesquels il est question de trouver ce difficile équilibre dans la répartition des biens. Papineau a réussi à l'atteindre, en s'inspirant de ce que le XVIII ${ }^{\mathrm{e}}$ siècle avait de plus généreux. Il ne pouvait faire autrement. Son bonheur en dépendait ${ }^{88}$.

${ }^{88}$ Dans cette étude, nous n'avons pas tenté de narrer l'évolution intellectuelle et religieuse de Papineau. Nous renvoyons le lecteur intéressé à l'étude de Lionel Groulx, intitulée "Les idées religieuses de Louis-Joseph Papineau", dans Notre maître, le passé (Montréal, Granger, 1936, 2: 167-211). Ce travail est toujours valable quoiqu'il ait été fait dans une optique cléricale et que la documentation sur la formation de Papineau ait été incomplète; car le chanoine Groulx n'a pas réussi à reconstituer le catalogue des ouvrages lus par Papineau durant la période de ses études. 